Check for updates

Cite this: RSC Adv., 2017, 7, 25694

Received 14th January 2017 Accepted 28th April 2017

DOI: $10.1039 / \mathrm{c} 7 \mathrm{ra00597k}$

rsc.li/rsc-advances

\section{Amphiphilic copolymer and TPGS mixed magnetic hybrid micelles for stepwise targeted co-delivery of DOX/TPP-DOX and image-guided chemotherapy with enhanced antitumor activity in liver cancer}

\author{
Xiaoju Shi, ${ }^{a}$ Guoyue Lv, ${ }^{a}$ Xiaodong Sun, ${ }^{a}$ Dianbo Cao, ${ }^{c}$ Guangyi Wang*a \\ and Yulei Chang (D)*b
}

\begin{abstract}
Recently, several targeted drug delivery systems have shown promising antitumor activity towards different types of cancer. However, targeting the tumor microenvironment, cellular surface and/or only one organelle may not achieve the expected therapeutic effects. Herein, a targeted drug delivery with stepwise targeting and image-guided strategy was obtained for enhancing antitumor therapy and overcoming various problems caused by the random drug distribution of traditional medicine. In this design, a multifunctional nanoplatform, TPGS/PPG2L@IONPs@DOX/TPP-DOX, based on poly(ethylene glycol)-poly(e-caprolactone)-poly(amidoamine)-lactose acid (mPEG-PCL-G2.0 PAMAM-LA, PPG2L) copolymer and $D-\alpha$-tocopherol polyethylene glycol 1000 succinate (TPGS), as a co-carrier, incorporates iron oxide nanoparticles (IONPs) as imaging agents and loads a DOX/triphenylphosphonium (TPP)-DOX drug for chemotherapy. In this nanoplatform micelle, the LA as targeting moieties to hepatocarcinoma cells is the first targeting step. After the drug is released from the hybrid micelles, the incorporated DOX and TPP-DOX target delivery to nuclei and mitochondria for the next targeting step. Furthermore, with the help of MRI contrast agents of IONPs, the distribution of hybrid micelles was monitored. As a result, the hybrid micelles demonstrated enhanced antitumor cell activity in both HepG2 and HepG2/DOX cells. The results indicated that the multifunctional hybrid micelles showed great potential in enhancing antitumor activity under image-guided and stepwise targeted chemotherapy.
\end{abstract}

\section{Introduction}

In the past decades, a multifunctional nanoplatform with targeted and image-guided drug delivery was developed to improve therapeutic efficacy and minimize systemic toxicity of the chemotherapy agents in cancer therapy. ${ }^{1,2}$ Recently, there have been substantial efforts to develop an intracellular drug delivery nanoplatform targeting specific subcellular organelles for enhanced antitumor activity. ${ }^{3,4}$ However, targeting tumor microenvironment, cellular surface and/or only one organelle may not achieve the expected therapeutic effects. One solution selected by researchers is to simultaneously target multisubcellular organelles or structures. In particular, targeted

\footnotetext{
${ }^{a}$ Department of Hepatobiliary \& Pancreatic Surgery, The First Hospital of Jilin University, Changchun, Jilin 130021, China. E-mail: wgymd@sina.com

${ }^{b}$ State Key Laboratory of Luminescence and Applications, Changchun Institute of Optics, Fine Mechanics and Physics, Chinese Academy of Sciences, Changchun, Jilin 130033, China. E-mail: yuleichang@ciomp.ac.cn

'Department of Radiology, The First Hospital of Jilin University, Changchun, Jilin 130021, China

$\dagger$ Electronic supplementary information (ESI) available. See DOI: $10.1039 / \mathrm{c} 7 \mathrm{ra00597k}$
}

drug delivery with stepwise targeting and image-guided strategy will be optimal for enhancing antitumor therapy. Compared with conventional therapeutics, nanoparticle-based formulations are larger in size, which allows them to preferentially accumulate in solid tumors through the enhanced permeability and retention (EPR) effect. ${ }^{5}$ The imaging agents are introduced into nano-formulations. On one hand, it will be beneficial to trace the accumulation in the tumor and the intratumoral distribution of nano-formulations for theranostics. On the other hand, it can be used as a carrier for drug delivery. Iron oxide nanoparticles (IONPs) have been employed as multidrug carriers for drug delivery and as magnetic resonance imaging (MRI) agents owing to the suitable size, outstanding magnetic properties and non-toxicity. ${ }^{6,7}$ To date, there are about five types of IONPs approved by the FDA and/or European Commission (EC) for MRI or iron supplement. ${ }^{8}$ Thus, the combination of diagnostics and therapeutics in one targeting system will improve drug delivery efficiency and biological safety to avoid the reticuloendothelial system (RES) during the antitumor therapy.

Doxorubicin (DOX) is an anthracycline chemotherapeutic drug that is widely used in antitumor therapy. Nuclear DNA damage is the predominant mechanism of DOX for direct 
anticancer effects. ${ }^{9}$ Recently, it has been found that in addition to the nucleus, mitochondrion is another vital organelle for targeting. This is because mitochondrial DNA can be damaged, resulting in the increase of reactive oxygen species (ROS), which results in the dysfunction of mitochondria and the decrease of ATP production. ${ }^{\mathbf{1 0 - 1 2}}$ Because mitochondrial metabolism is critical for the survival of cancer cells and even drug resistant cells, mitochondrial drug delivery may prove to be an important strategy for anticancer therapy. ${ }^{13}$ Therefore, the simultaneous delivery of DOX to the nucleus and mitochondria can increase the antitumor activity. Very recently, a few mitochondria targeting agents have successfully been applied to target DOX to mitochondria., ${ }^{3,13,14}$ However, the severe cardiac toxicity problems and the multidrug resistance (MDR) effect of DOX remain to be overcome. Stepwise targeting cell surface and subcellular organelles under the image-guided combination strategy are supposed to achieve a high efficiency and biosafety in chemotherapy. To further overcome MDR, the TPGS was a good candidate as the carrier to inhibit P-gp-mediated MDR via influencing the activity of P-gp ATPase..$^{15,16}$ TPGS has been accepted by the FDA as a pharmaceutical solubilizer and adsorption enhancer for several poorly water-soluble drugs. ${ }^{17,18}$ To achieve the stability of a drug loading system, it is generally mixed with other amphiphilic materials for drug encapsulation. ${ }^{\mathbf{1 9 2 0}}$

In this study, an MRI image-guided multifunctional nanoplatform was designed for co-delivery of DOX/TPP-DOX, which was prepared by LA-modified PAMAM dendrimer based copolymer and TPGS (Scheme 1). The cellular internalization of the LAmodified hybrid micelles is expected to be selectively delivered to the HepG2 cells for the first step due to high-affinity of LA for the asialoglycoprotein receptor (ASGP-R) on the membrane of HepG2 cells $^{\mathbf{2 1 , 2 2}}$ and the dual subcellular organelle targeting strategy for a further step, allowing the enhancement of antitumor activities. The mitochondrial targeting of TPP-DOX is ascribed to the high lipophilicity and stable cationic charge of TPP; it can be taken up by the mitochondrial membrane after attaching TPP to drug molecules or a carrier. ${ }^{13,23}$ Furthermore, with the help of MR imaging agents of IONPs, the accumulation of the drugs in the tumor, the intratumoral distribution, and the kinetics of drug release are monitored in real time, which will be a part of the very early step in the practice of precision medicine.

\section{Experimental}

\subsection{Materials}

Doxorubicin (DOX) was purchased from Dalian Meilun. $\mathrm{N}$-(3Dimethylaminopropyl)- $N$-ethyl-carbodiimide hydrochloride (EDCI) and 1-(4,5-dimethylthiazol-2-yl)-3,5-diphenylformazan (MTT) were purchased from Sigma Aldrich. TPGS was obtained from Aladdin. (3-Carboxypropyl)triphenylphosphonium bromide (TPP) was obtained from TCI. 5,5' ,6,6'-Tetrachloro$1,1^{\prime}, 3,3^{\prime}$-tetraethylbenzimidazolylcarbocyanine iodide (JC-1), Hoechst 33342 and Mito-tracker were obtained from Beyotime Biotechnology. Iron oxide nanoparticles (IONPs) with oleic acid (OA) coating were obtained from Ocean NanoTech. All other reagents were of analytical grade and used as received. Dendrimer based mPEG-PCL-G2.0 PAMAM (PPG2) copolymer was synthesized and characterized in our previous study. ${ }^{24}$ HepG2 cells and A549 cells were obtained from the College of Basic Medical Sciences, Jilin University. The HepG2/DOX cells were purchased from AIYAN Biological Technology Co., LTD., Shanghai, China. RPMI 1640 medium and fetal bovine serum (FBS) were obtained from Gibco. Deionized water from a Milli-Q device (18.2 MU; Millipore) was used throughout this study.

\subsection{Synthesis of TPP-DOX}

The prodrug of TPP-DOX was prepared by EDCI coupling reaction. Briefly, DOX base was obtained by adding triethylamine

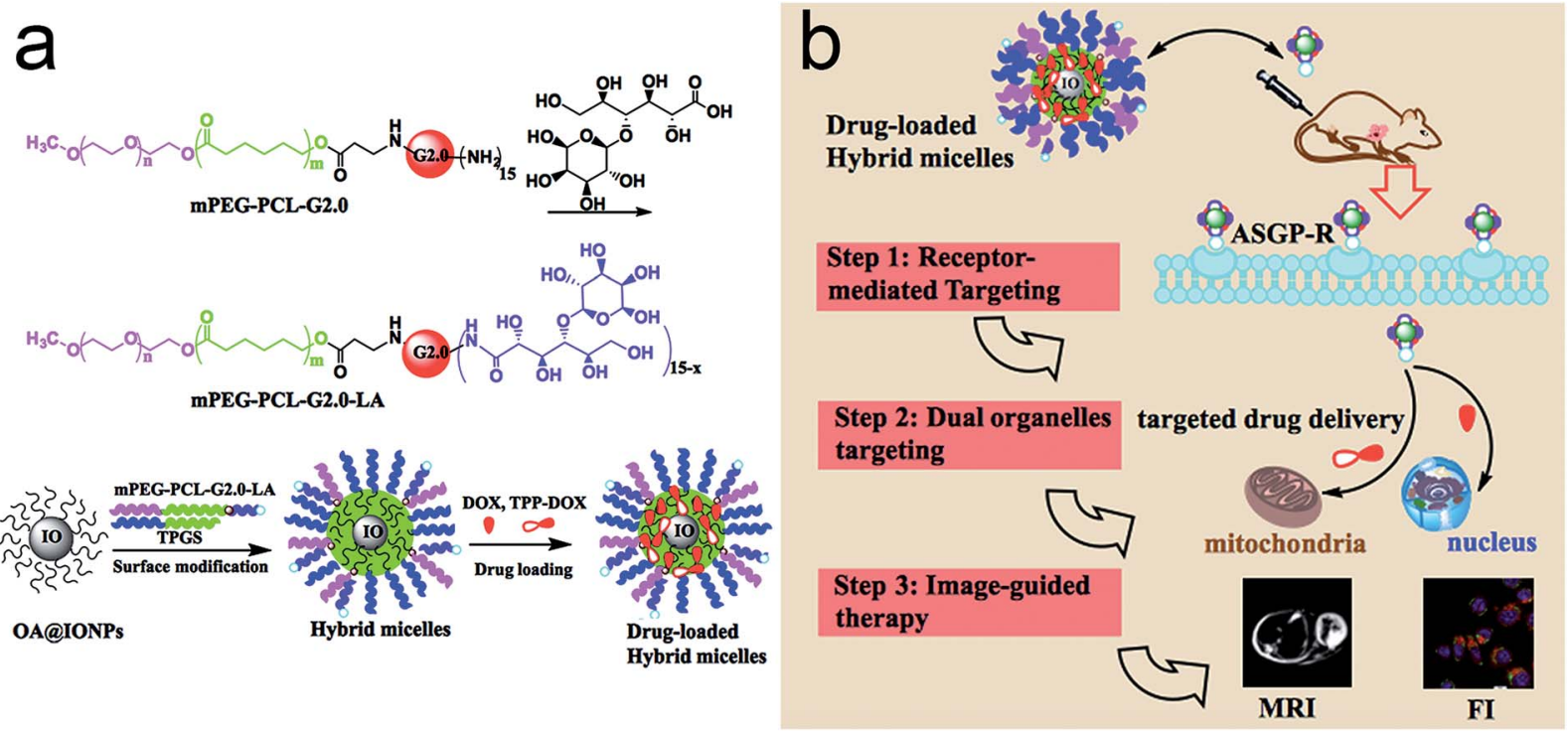

Scheme 1 (a) Synthetic routes employed for the preparation of hybrid micelles for drug-loading via hydrophobic interaction with TPGS/PPG2L mixed polymer with OA-capped IONPs (b) and its stepwise-targeted drug delivery and image-guided chemotherapy. 
( $5 \mu \mathrm{L}, 37 \mu \mathrm{mol}$ ) dropwise to the $5 \mathrm{~mL}$ DMSO solution containing $20 \mathrm{mg}$ of DOX $\mathrm{HCl}\left(4.0 \mathrm{mg} \mathrm{mL} \mathrm{mb}^{-1}\right)$ while stirring. The supernatant of EDCI activated $15 \mathrm{mg}$ of $\mathrm{COOH}-\mathrm{TPP}$ was then added dropwise to the above DOX base solution. The mixture was stirred overnight at room temperature (RT) without illumination. The crude product was precipitated by ether and purified by flash chromatography. The synthesized TPP-DOX molecule was characterized by ${ }^{1} \mathrm{H}$ NMR and Fourier transform infrared (FT-IR) spectroscopy.

\subsection{Synthesis of mPEG-PCL-G2.0 PAMAM-LA (PPG2L)}

For the conjugation of LA, $96 \mathrm{mg}$ of PPG2L and $9.8 \mathrm{mg}$ of LA were dissolved in $3 \mathrm{~mL}$ DMSO. After $11 \mathrm{mg}$ EDCI was added, the mixture was stirred at RT overnight. The mixture solution was dialyzed with a dialysis tube (cut-off $\mathrm{Mw}$ : $3.5 \mathrm{kDa}$ ) for 2 days to remove free LA. After that, the obtained solution was lyophilized for further use. The synthesized copolymer was fully characterized by ${ }^{1} \mathrm{H}$ NMR and FT-IR spectroscopy in ESI.

\subsection{Preparation of hybrid micelles}

The PPG2L copolymer and TPGS mixed micelles were prepared by an equilibrium dialysis method as previously reported. ${ }^{24}$ The as-obtained PPG2L (40.0 mg) and TPGS (40.0 mg) were well dissolved in $2.0 \mathrm{~mL}$ of tetrahydrofuran (THF). Another $2.0 \mathrm{~mL}$ of IONP THF solution ( $2 \mathrm{mg} \mathrm{mL}^{-1}$ ) was added to the above solution, followed by the addition of equal volume of water drop by drop. The mixture solution was then transferred into a dialysis bag (cut-off $3.5 \mathrm{kDa}$ ) and dialyzed against water with solvent changed every $4 \mathrm{~h}$ for 2 days. Subsequently, the micelles were purified through centrifugation to remove the excess free polymer and filtered with a $0.22 \mu \mathrm{m}$ filter and finally dried through lyophilization. The particle sizes of mixed micelles were determined by dynamic light scattering (DLS) method using a Zetasizer Nano ZS (Malvern Instruments, Malvern, UK). For the analysis of micelle morphology, transmission electron microscopy (TEM) was used.

\subsection{Drug loading and release profiles of hybrid micelles}

The hybrid micelles were decorated with DOX/TPP-DOX by soaking the drug mixtures ( $2 \mathrm{mg}$ DOX and $2 \mathrm{mg}$ TPP-DOX) in an aqueous solution $(4 \mathrm{~mL})$ in a $10 \mathrm{~mL}$ round bottom flask and stirring overnight at RT. The formed reddish composite drugloaded hybrid micelles were collected by filtration (microfiltration membrane: $0.45 \mu \mathrm{m}$ ) and centrifugation $(11000 \mathrm{rpm}$ for $15 \mathrm{~min}$ ) and then washed with water 3 times, followed by lyophilization. Similarly, the hybrid micelle for only entrapping the drug was prepared using the same method. The loading efficiency (\%) was calculated by the ratio of measured and initial amount of DOX solubilized in the supernatant, which was defined as the amount of DOX/TPP-DOX (mg) loaded for $100 \mathrm{mg}$ of hybrid micelles. The DOX/TPP-DOX ratio was measured by high performance liquid chromatography (HPLC, COSMOSIL C18) analysis of the supernatant, which was collected after centrifugation. Mobile phase was acetonitrile/ water $(0.5 \%$ formic acid $)=90: 10(\mathrm{v} / \mathrm{v})$, and the flow rate was $0.3 \mathrm{~mL} \mathrm{~min}^{-1}$. Analysis was performed at RT. Injection volume was kept at $20 \mu \mathrm{L}$. The drug release profiles of hybrid micelles were assessed by the dialysis method against different $\mathrm{pH}$ buffer solutions ( $\mathrm{pH} 7.4$ and 5.0) at $37{ }^{\circ} \mathrm{C}$. Briefly, $2 \mathrm{~mL}$ of the drugloaded micelle solution in the dialysis membrane (cut-off $3500 \mathrm{kDa}$ ) was submerged in $20 \mathrm{~mL}$ of buffer at $37^{\circ} \mathrm{C}$. At predetermined time intervals, $1.0 \mathrm{~mL}$ dialysis solution was taken out and detected by a UV-Vis spectrophotometer. After that, the solution was immediately returned back to the original release system.

\subsection{Cytotoxicity assay}

HepG2 and HepG2/DOX cells were used in this study. Take HepG2 cells as an example. Briefly, HepG2 cells were cultured in 1640 medium supplemented with $10 \%$ fetal bovine serum and maintained in a humidified atmosphere with $5 \% \mathrm{CO}_{2}$ under $37{ }^{\circ} \mathrm{C}$. For the MTT cytotoxicity assay, the suspended cells at a concentration of $1 \times 10^{5} \mathrm{~mL}^{-1}$ were planted in a 96-well plate with $100 \mu \mathrm{L}$ per well. After $24 \mathrm{~h}$ incubation for full attachment and stabilization, HepG2 cells were treated with fresh medium containing different as-prepared hybrid micelles with various concentrations. After $24 \mathrm{~h}$ incubation in the dark, the cell viability was evaluated by the standard MTT assay. The cell viability was quantified by measuring the absorbance at $490 \mathrm{~nm}$ using a microplate reader (BioTech808).

\subsection{Cellular uptake and subcellular distribution}

For improving the therapeutic effects of DOX in chemotherapy, the cellular internalization of the LA-modified hybrid micelles is expected to be selectively delivered to the tumor cells for the first step and the dual subcellular organelle targeting strategy for further steps. The cellular uptake profiles were studied by confocal laser scanning microscopy (CLSM) via fluorescence imaging. The HepG2 cells were seeded onto a glass bottom disk at a density of $1.5 \times 10^{4}$ cells per well and cultured at optimum conditions. After the attachment of HepG2 cells for $24 \mathrm{~h}$, the equivalent DOX concentrations of free DOX, TPP-DOX and asprepared micelles $\left(2 \mu \mathrm{g} \mathrm{mL}^{-1}\right)$ were added and incubated for another $4 \mathrm{~h}$. The culture medium was replaced and washed several times with phosphate-buffered saline (PBS). Hoechst 33342 and Mito-tracker green were used to stain the nuclei and mitochondria according to the manufacturer's specification. A Nikon microscope was used to investigate the intracellular colocalization of DOX/TPP-DOX on subcellular organelles.

\subsection{Mitochondrial membrane potential determination}

JC-1 is a specific dye of mitochondrial membrane potential (MMP, $\Delta \Psi_{\mathrm{m}}$ ), which is used to evaluate the alterations in $\Delta \Psi_{\mathrm{m}}$. The detection principle is the decrease of membrane potential JC-1 emission light from red to green, indicating mitochondrial membrane damage. Taking HepG2 cells as an example, in brief, HepG2 cells $\left(1 \times 10^{5}\right.$ cells per well $)$ were seeded onto 6-well plates for $24 \mathrm{~h}$. The DOX/TPP-DOX hybrid micelles and DOX micelles were added and incubated for $4 \mathrm{~h}$ in the same way. After that, the treated cells were washed three times with PBS and incubated in complete culture medium containing JC-1 dye $\left(1.0 \mu \mathrm{g} \mathrm{mL}{ }^{-1}\right)$ for $20 \mathrm{~min}$ at $37{ }^{\circ} \mathrm{C}$. Then, the harvested and 
dispersed cells in solution were detected using a fluorescent spectrophotometer. Red-to-green ratios were calculated from the corresponding fluorescence intensity.

\subsection{Animal modal and in vivo MRI}

Animal experiment was carried out strictly in accordance with governmental and international guidelines of the Ministry of Science and Technology of the China's Guide for the Care and Use of Laboratory Animals and approved by the Animal Care and Use Committee of Jilin University. Tumor-bearing mice were prepared by the subcutaneous injection of $100 \mu \mathrm{L}$ of $\mathrm{H} 22$ cells in female ICR mice (about $20 \mathrm{~g}$, Jilin University Animal Center, SCXK-2008-0004). We performed MRI experiments according to the method reported previously. ${ }^{24}$ The tumor-bearing mice were randomly divided into 2 groups ( $n=3$ per group), one of which was injected intratumorally with $100 \mu \mathrm{L}$ of saline, and the others were injected with as-obtained hybrid micelles with the same volume. MRI was performed using a $1.5 \mathrm{~T}$ Siemens Avanto MR scanner with an animal coil by one radiologist at $3 \mathrm{~h}$ and $24 \mathrm{~h}$ post-injection. The signal to noise ratio (SNR) was calculated as follows: $\mathrm{SNR}=\mathrm{SI}$ (tumor)/SD (noise), which was defined by signal intensity (SI) and standard deviation (SD).

\section{Results and discussion}

\subsection{Construction of hybrid micelles}

The successful synthesis of mitochondria targeting prodrug of TPP-DOX and amphiphilic copolymer of PPG2L was characterized by ${ }^{1} \mathrm{H}$ NMR and FT-IR, respectively, as shown in Fig. S1$\mathrm{S} 4$ (see ESI $\dagger$ for details). The drug-loaded hybrid micelles are schematically illustrated in Scheme 1.

The PPG2L and TPGS self-assembled with OA-IONPs were prepared by hydrophobic-hydrophobic interactions, forming a hydrophobic layer on the IONP surface that provides active adsorption sites to load lipophilic drug molecules of TPP-DOX/ DOX also via hydrophobic interactions. The formation of PPG2L and TPGS mixed hybrid micelles was characterized by the changes of hydrodynamic diameters. The main size of TPGS/ PPG2L@IONPs hybrid micelles was about $26.2 \pm 3.5 \mathrm{~nm}$, which fell into the window of PPG2L@IONPs mono-micelles $(37.12 \pm 7.9 \mathrm{~nm})$ and TPGS@IONPs mono-micelles (15.06 \pm $4.05 \mathrm{~nm})$. The results above indicate the formation of mixed polymer hybrid micelles rather than the mixture of two monopolymer hybrid micelles. ESI Table $1 \uparrow$ summarizes the other related nanoparticle sizes, and polydispersity index (PDI) was determined by dynamic light scattering (DLS) in ESI. The morphology and size distribution of as-prepared hybrid micelles were also measured by transmission electron microscopy (TEM). Under TEM as shown in Fig. 1, compared with the OA-capped IONPs (Fig. 1a), the IONPs in the hybrid micelles showed the same size and morphology as before, and an organic layer around IONPs indicated the formation of hybrid micelles as shown in Fig. 2b. The average size was about $16.2 \pm 3.8 \mathrm{~nm}$, which was slightly smaller than that of DLS because TEM only exhibits the size of dried structures. Furthermore, the surface morphology of the as-obtained micelles has been analyzed by

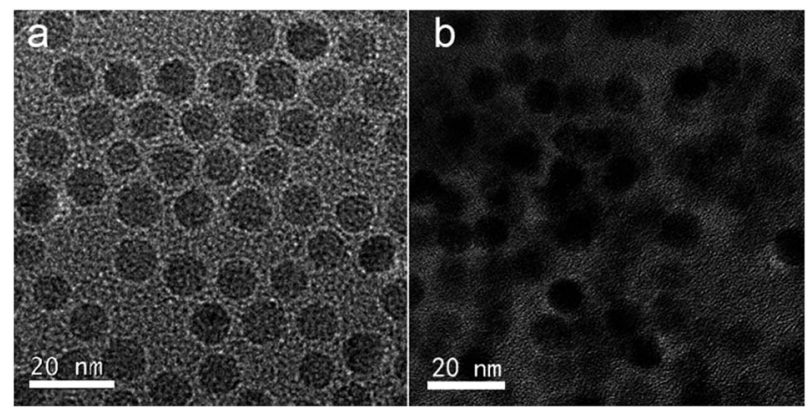

Fig. 1 TEM images of (a) OA-capped IONPs, and (b) drug-loaded hybrid micelles. Scale bar is $20 \mathrm{~nm}$.

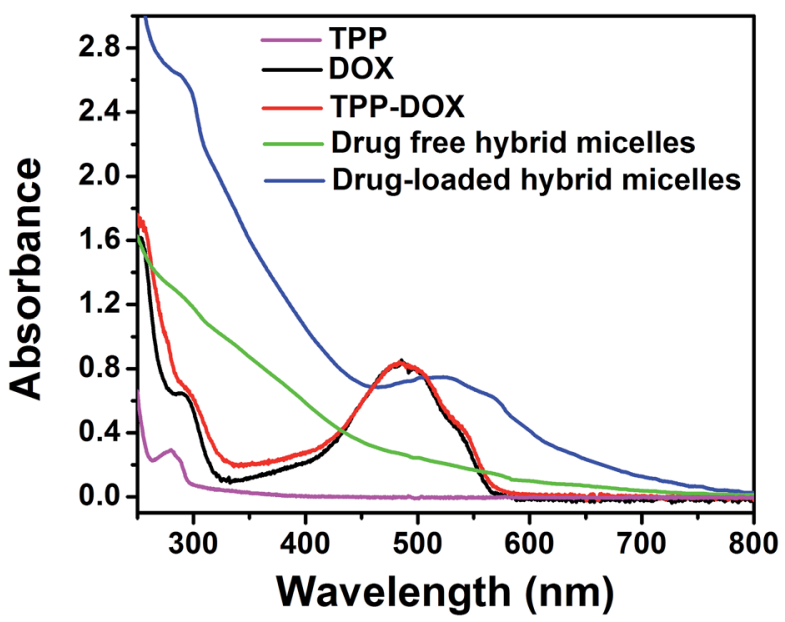

Fig. 2 UV-Vis absorption spectra of TPP in pink, free DOX in black, TPP-DOX in red, drug free hybrid micelles in green and drug-loaded hybrid micelles in blue.

AFM, as shown in Fig. S5 (ESI $\dagger$ ). The successful surface modification of OA-IONPs could also be confirmed by the results of phase-transfer from the original organic phase to the aqueous medium. All these results confirmed the formation of hybrid micelles. To investigate drug-loading efficiency, the as-prepared hybrid micelles of TPGS/PPG2L@IONPs were impregnated within TPP-DOX/DOX mixed solutions under stirring. After $24 \mathrm{~h}$ interaction, any unloaded free drug was removed by filtration and centrifugation. Compared with free DOX, TPP-DOX and TPGS/PPG2L@IONPs, a characteristic absorption peak of DOX around $490 \mathrm{~nm}$ was observed apparently from UV-Vis spectra (Fig. 2), and the drug loading capacity was evaluated by calculating the UV-Vis absorbance change of the supernatant. At the same time, the formation of TPP-DOX was confirmed by the UVVis by comparing the TPP and DOX absorption spectra. The total drug loading capacity was $8.4 \%(\mathrm{w} / \mathrm{w})$ calculated from the UV-Vis (on the basis of DOX). The DOX/TPP-DOX mass ratio was 0.6, which was obtained by analysis of the supernatant via HPLC. The typical HPLC spectra of DOX and TPP-DOX are shown in Fig. S6. $\dagger$ Both of the hybrid micelles are stable in PBS without size changes, and there is no IONPs leaking out over two months. It indicated that this type of hybrid micelle was a satisfactory drug delivery and imaging system. 


\subsection{In vitro drug release}

The TPP-DOX/DOX release profiles of hybrid micelles in $48 \mathrm{~h}$ against different $\mathrm{pH}$ buffers are shown in Fig. S7. $\uparrow$ The normal physiological conditions of $\mathrm{pH} 7.4$ and intracellular acid conditions around pH 5.0, for lysosomes and endosomes, were selected to simulate the physiological conditions. It was found that the cumulative release rates of DOX and TPP-DOX from the hybrid micelles showed a $\mathrm{pH}$-dependence. Lowering the $\mathrm{pH}$ leads to the different release profiles at pH 5.0 (about 60\%) and pH 7.4 (about $28 \%$ ). The weakening of hydrophobic interaction is mainly because the hydrophilicity of DOX in micelles being enhanced with the decrease in $\mathrm{pH}$. It indicates that the encapsulated drug of DOX and TPP-DOX in the hybrid micelles would be rapidly released under acidic environment of intracellular acidic organelles (e.g., lysosome/endosome) of cancer cells. After that, the released DOX/TPP-DOX is conducive to targeting the nucleus and mitochondria for enhanced antitumor activity. The DOX release profiles from only DOX-loaded hybrid micelles were similar to those of DOX (as shown in Fig. S3b†). After the drug release from the drug-loaded hybrid micelles against different $\mathrm{pH}$ buffer solution, we also found that there was no hybrid micelle disruption, which led to the IONPs' leakage. All the results indicated that the hybrid micelles are good carriers for drug loading and delivery.

\subsection{Cell uptake and subcellular distribution profiles}

To evaluate the intracellular localization of prodrug (DOX/TPPDOX) delivered by drug-loaded hybrid micelles, we performed a colocalization study using subcellular organelle staining. The yellow and purple spots in the merged images demonstrate the overlap of green (Mito-tracker green) with red (TPP-DOX) and blue (nuclei are stained by Hoechst 33342) with red (DOX) fluorescence, respectively. The CLSM images of HepG2 cells stained for nuclei and mitochondria are shown in Fig. 3. It can be seen that the nuclei and mitochondria showed the DOX's (or TPP-DOX) red fluorescence, which were the targets of DOX and TPP-DOX, respectively. Our results demonstrated that TPGS/PPG2L@IONPs@DOX/TPPDOX hybrid micelles could effectively deliver DOX and TPP-DOX to the nucleus and mitochondria, respectively, which resulted in high cytotoxicity of as-prepared micelles.

\subsection{The effect of TPGS/PPG2L@IONPs@DOX/TPP-DOX on the mitochondrial membrane potential}

As known, the mitochondrion plays the most prominent roles in producing the energy currency of the cell and regulating cellular metabolism. Although the characteristic structural alterations of apoptosis are mainly in the nucleus, it is now clear that mitochondria are key organelles in the programmed death signal transduction pathway. ${ }^{25,26}$ The attenuation of MMP is usually accompanied with the release of cytochrome c (Cyto-c) and the activation of caspase- 9 and caspase- $3 .^{27}$ Therefore, delivery of DOX to mitochondria could promote the tumor cell apoptosis. The as-prepared DOX only hybrid micelles (TPGS/ PPG2L@IONPs@DOX) and DOX/TPP hybrid micelles (TPGS/ PPG2L@IONPs@DOX/TPP-DOX) on MMP of HepG2 cells and HepG2/DOX cells were probed using JC-1 dye staining as shown

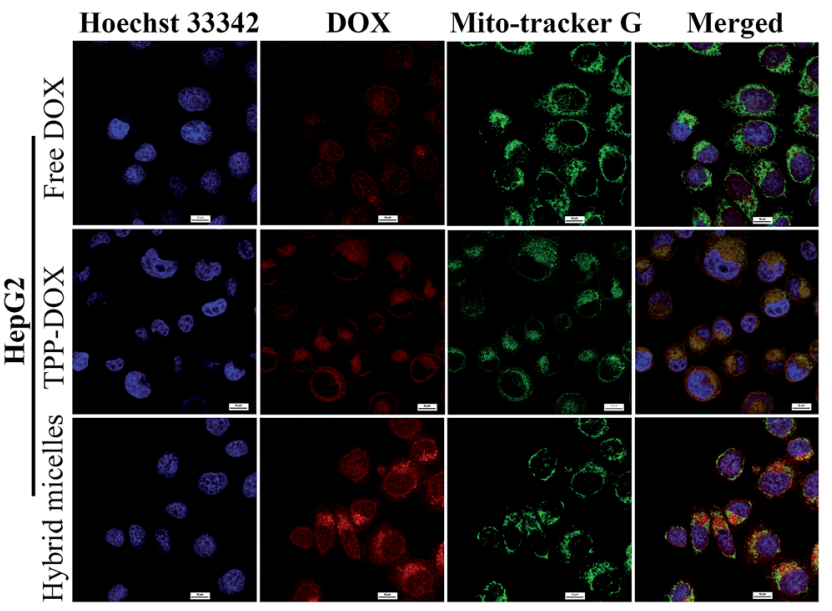

Fig. 3 Intracellular distribution of free DOX, TPP-DOX and drugloaded hybrid micelles in HepG2 cells. Cells were exposed to corresponding solution at $37^{\circ} \mathrm{C}$ for $24 \mathrm{~h}$, and the intracellular mitochondria were stained by Mito-Tracker Green followed by nucleus staining with Hoechst 33342. The cells were observed under CLSM.

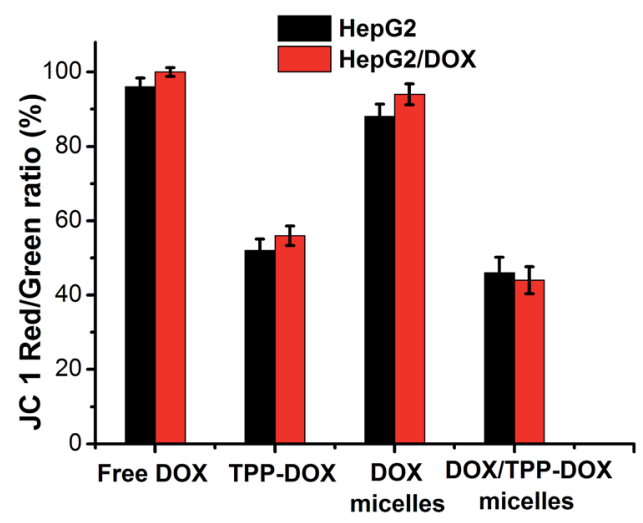

Fig. 4 The effect of as-prepared drug-loaded hybrid micelles on the MMP of HepG2 cells and HepG2/DOX cells. The equivalent DOX concentration of free DOX, TPP-DOX and as-prepared micelles $\left(2 \mu \mathrm{gL}^{-1}\right.$ ) was used. Data are presented as mean $\pm \mathrm{SD}, n=3$.

in Fig. 4. The decrease of red-to-green intensity ratio illustrates depolarization of mitochondria. As can be seen, the red-togreen ratio was not significant in reduction when HepG2 cells were treated with DOX only micelles. On the contrary, the redto-green ratio significantly decreased when HepG2 cells were cultured with DOX/TPP-DOX micelles. Similar results were observed for HepG2/DOX cells. The above results indicated that TPP-mediated DOX targeted delivery to mitochondria caused the decrease of MMP, which can not only cause cell apoptosis, but also affect the production of ATP, whereas P-gp is an ATPdependent drug efflux pump. ${ }^{28}$ Accordingly, the reduction of MMP may lead to the decrease of P-gp-mediated drug efflux and the increase of the drug accumulation in the drug resistant tumor cells, subsequently enhancing the antitumor activity.

\subsection{In vitro cytotoxicity and anticancer activity}

Now we turn to the cytotoxicity of drug-loaded hybrid micelles against HepG2 and HepG2/DOX cancer cells. Cells above were 
used to evaluate dark cytotoxicity of the drug-loaded free hybrid micelles. There were no significant differences among concentrations from $0.1 \mathrm{mg} \mathrm{mL}^{-1}$ to $1.2 \mathrm{mg} \mathrm{mL}^{-1}$. At $0.8 \mathrm{mg} \mathrm{mL}^{-1}$, all the cell viabilities were above $90 \%$ for up to $48 \mathrm{~h}$ (Fig. $5 \mathrm{a}$ and b). Following the outcome, these cells were incubated for $48 \mathrm{~h}$ with the various DOX-based formulations at different concentrations $\left(0.25,0.5,1.25,2.5,5.0\right.$ and $\left.10.0 \mu \mathrm{g} \mathrm{mL}{ }^{-1}\right)$. The enhanced antitumor efficacy was evaluated by comparing with original DOX under the same condition as shown in Fig. 5c. Drug-loaded hybrid micelles and free DOX both showed dose-dependent cytotoxicity at $48 \mathrm{~h}$; however, a significant dependence and lower cytotoxicity were observed for HepG2 cells incubated with the hybrid micelles compared with free DOX. It is mainly due to the different cellular uptake profiles and sustained release of drug from the hybrid micelles as suggested in several studies. ${ }^{29-31}$ The high antitumor efficacy of LA-hybrid micelles toward HepG2 cells may be due to the enhanced cellular uptake attributed to ASGP receptor-mediated endocytosis, which was further confirmed by the A549 cell line without the ASGPreceptor. The drug-loaded LA-hybrid micelles and nontargeting hybrid micelles on A549 cells showed no significant difference of cytotoxicity, as shown in Fig. S9. $\dagger$ Moreover, to assess the potential of the as-prepared TPGS-based hybrid micelles (TPGS/PPG2L@IONPs-DOX and TPGS/PPG2L@IONPsDOX/TPP-DOX) for MDR reversal in HepG2/DOX antitumor treatment, we evaluated the cell viabilities as treated with TPGSbased hybrid micelles and TPP-DOX containing hybrid micelles by MTT assay as shown in Fig. $5 d$. It is shown from the results that DOX-loaded hybrid micelles at high concentration showed significantly enhanced cytotoxicity for $48 \mathrm{~h}$, whereas free DOX had no significant difference in cell viability, further indicating that TPGS played a role in reversing MDR. Notably, the TPGS/ PPG2L@IONPs@DOX/TPP-DOX micelles had a high antitumor efficacy compared to that of the DOX-loaded only hybrid micelles. It indicated that the targeted drug delivery to mitochondria could effectively overcome MDR.

\subsection{In vivo MR image-guided therapy}

The drug-loaded hybrid micelles consisted of imaging contrast agents, which allowed detecting the disease for diagnosis and directly monitoring its current distribution state. Furthermore, MRI as one of the most powerful non-invasive clinical imaging techniques is widely used in clinical diagnosis. After the TGA analysis, the corresponding hybrid micelles contain $41 \%$ of IONPs by weight. This data was also given in the ESI as Fig. S8. $\dagger$ Thus, as a proof of principle experiment, we explored IONPs as a T2-weighted MRI agent for in vivo tumor detection, as well as drug distribution probes in Fig. 6a. For the groups injected with IONP-based hybrid micelles, we could see clearly enhanced quality of MR images of tumor sites relative to control group mice injected with saline (front) at $3 \mathrm{~h}$ post-injection (middle). The darker sites of MR images could also show the drug distribution in the tumor. The SNR decreased significantly
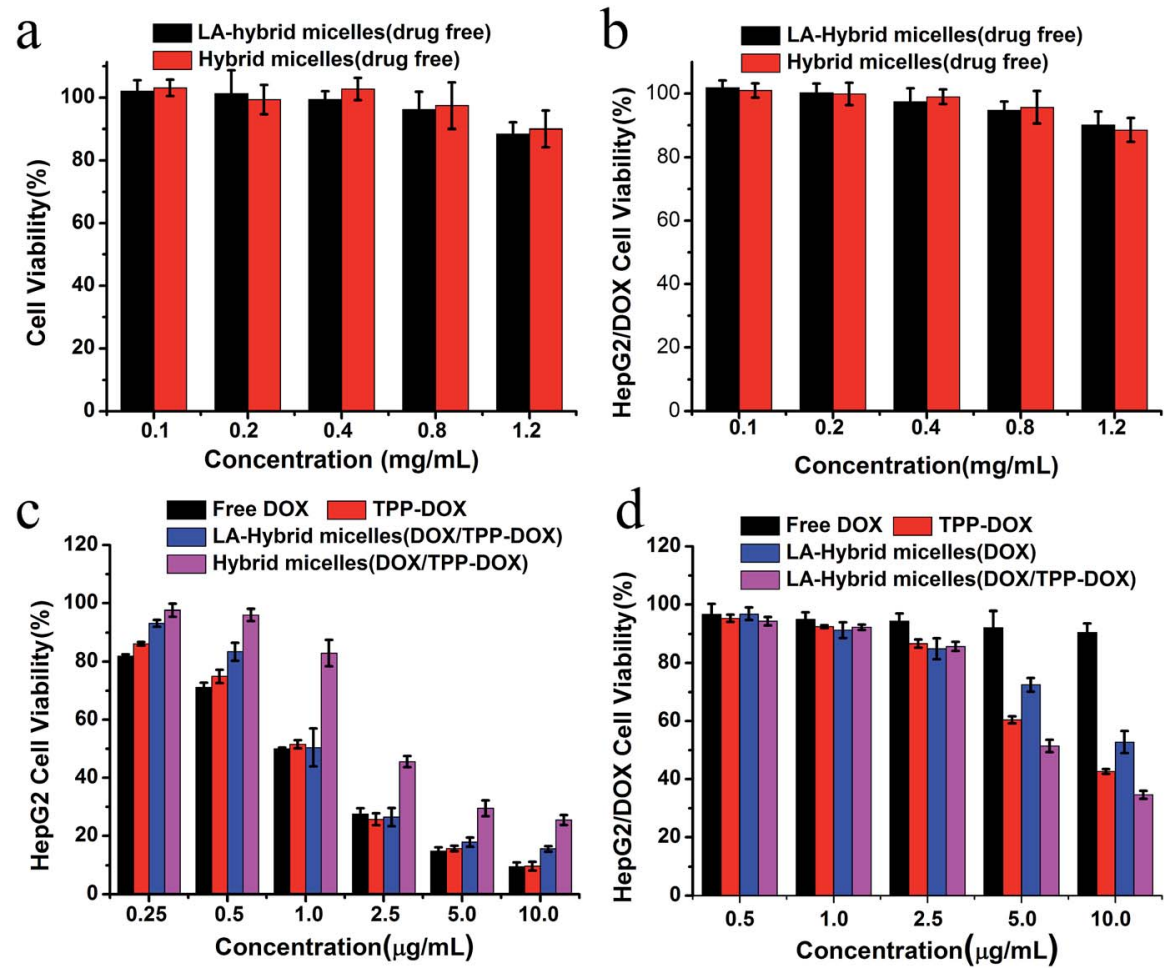

Fig. 5 The dark cytotoxicity of LA hybrid micelles (TPGS/PPG2L(aIONPs) and hybrid micelles (TPGS/PPG2@IONPs) on HepG2 cells (a) and HepG2/DOX cells (b) for $48 \mathrm{~h}$ at $37^{\circ} \mathrm{C}$. The cytotoxicity of (c) free DOX and drug-loaded hybrid micelles with or without LA at different concentrations on HepG2 cells for $48 \mathrm{~h}$, (d) free DOX and drug-loaded hybrid micelles with or without TPP-DOX on HepG2/DOX for $48 \mathrm{~h}$. The cells were exposed to DOX or TPP-DOX at equivalent concentrations. Data are presented as mean \pm SD $(n=3)$. 


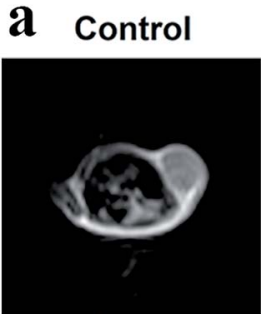

b

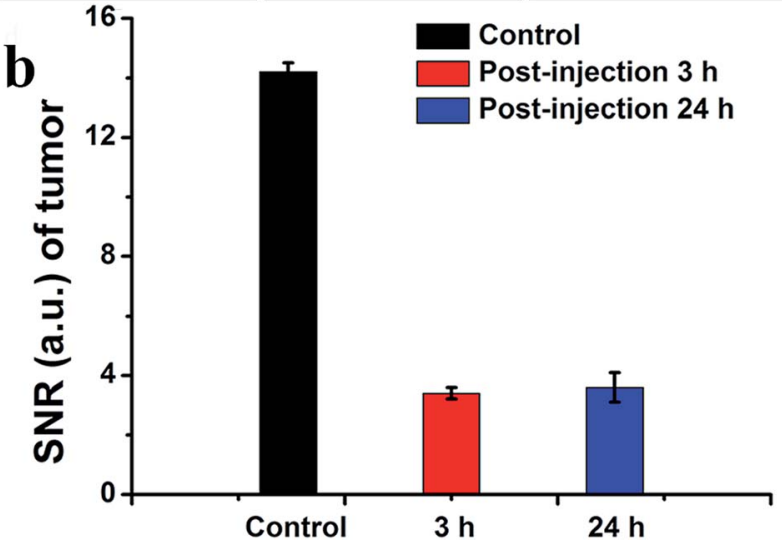

Fig. 6 (a) T2-MRI of tumor-bearing mice for determined time intervals post-intratumoral injection of LA-hybrid micelles after $3 \mathrm{~h}$ and $24 \mathrm{~h}$, with the saline group as control and (b) the corresponding SNR.

following intratumoral injection of hybrid micelles (Fig. 6b). SNR was $14.2 \pm 0.3$ injection of saline and $3.4 \pm 0.2$ (postinjection $3 \mathrm{~h}$ ). We next evaluated the distribution of localized drugs at $24 \mathrm{~h}$ post-injection. The SNR measurements were $3.6 \pm$ 0.5. Compared with the situation at $3 \mathrm{~h}$, the localized drug slowly spread in the tumor tissues, which proved essential information for theranostics.

\section{Conclusion}

In summary, we reported the in vitro and in vivo evaluation of a stepwise-targeted drug delivery and image-guided approach by exploring hybrid micelles as a delivery nanosystem, which is capable of delivery to cellular membranes and subcellular organelles for enhancing therapeutic effects. The results further demonstrated that the drug-loaded hybrid micelles simultaneously targeted delivery to mitochondria and nucleus and exhibited good MRI contrast properties. Guided by the images of MRI, the intratumoral distribution of hybrid micelles was obtained for theranostics. We consider that this study offers an important step to explore the concept of image-guided and stepwise-targeted precise chemotherapy via specific organellerelated enhanced antitumor activity.

\section{Acknowledgements}

This study is financially supported by the Natural Science Foundation (NSF) of China (11174277, 11374297, 61275202, 21304084, 11474278 and 51372096) and the Foundation of Jilin province (20170520112JH).

\section{Notes and references}

1 H. J. Li, J. Z. Du, J. Liu, X. J. Du, S. Shen, Y. H. Zhu, X. Wang, X. Ye, S. Nie and J. Wang, ACS Nano, 2016, 10, 6753-6761.

2 F. Chen, H. Hong, Y. Zhang, H. F. Valdovinos, S. Shi, G. S. Kwon, C. P. Theuer, T. E. Barnhart and W. Cai, ACS Nano, 2013, 7, 9027-9039.

3 S. Imstepf, V. Pierroz, R. Rubbiani, M. Felber, T. Fox, G. Gasser and R. Alberto, Angew. Chem., 2016, 55, 2792-2795.

4 A. R. Maity and D. Stepensky, Mol. Pharmaceutics, 2016, 13, $1-7$.

5 Y. M. a. H. Maeda, Cancer Res., 1986, 46, 6387-6392.

6 Y. L. Chang, N. A. Liu, L. Chen, X. L. Meng, Y. J. Liu, Y. P. Li and J. Y. Wang, J. Mater. Chem., 2012, 22, 9594-9601.

7 Y. L. Chang, X. L. Meng, Y. L. Zhao, K. Li, B. Zhao, M. Zhu, Y. P. Li, X. S. Chen and J. Y. Wang, J. Colloid Interface Sci., 2011, 363, 403-409.

8 J. Huang, Y. Li, A. Orza, Q. Lu, P. Guo, L. Wang, L. Yang and H. Mao, Adv. Funct. Mater., 2016, 26, 3818-3836.

9 M. S. Chan, D. Y. Tam, Z. W. Dai, L. S. Liu, J. W. T. Ho, M. L. Chan, D. Xu, M. S. Wong, C. Tin and P. K. Lo, Small, 2016, 12, 770-781.

10 S. Marrache and S. Dhar, Proc. Natl. Acad. Sci. U. S. A., 2012, 109, 16288-16293.

11 M. Han, M. R. Vakili, H. Soleymani Abyaneh, O. Molavi, R. Lai and A. Lavasanifar, Mol. Pharmaceutics, 2014, 11, 2640-2649.

12 Q. Hu, M. Gao, G. Feng and B. Liu, Angew. Chem., Int. Ed., 2014, 53, 14225-14229.

13 M. Han, M. R. Vakili, H. S. Abyaneh, O. Molavi, R. Lai and A. Lavasanifar, Mol. Pharmaceutics, 2014, 11, 2640-2649.

14 T. A. Theodossiou, Z. Sideratou, M. E. Katsarou and D. Tsiourvas, J. Pharm. Res., 2013, 30, 2832-2842.

15 E.-M. Collnot, C. Baldes, M. F. Wempe, R. Kappl, J. Huettermann, J. A. Hyatt, K. J. Edgar, U. F. Schaefer and C.-M. Lehr, Mol. Pharmaceutics, 2007, 4, 465-474.

16 J. Wang, J. Sun, Q. Chen, Y. Gao, L. Li, H. Li, D. Leng, Y. Wang, Y. Sun, Y. Jing, S. Wang and Z. He, Biomaterials, 2012, 33, 6877-6888.

17 Y. Bao, M. Yin, X. Hu, X. Zhuang, Y. Sun, Y. Guo, S. Tan and Z. Zhang, J. Controlled Release, 2016, 235, 182-194.

18 F. Danhier, T. T. Kouhe, N. Duhem, B. Ucakar, A. Staub, N. Draoui, O. Feron and V. Preat, Int. J. Pharm., 2014, 476, 9-15.

19 Y. Mi, J. Zhao and S. S. Feng, Int. J. Pharm., 2012, 438, 98-106. 20 Y. Duan, B. Zhang, L. Chu, H. H. Tong, W. Liu and G. Zhai, Colloids Surf., B, 2016, 141, 345-354.

21 Y. Cao, Y. He, H. Liu, Y. Luo, M. Shen, J. Xia and X. Shi, J. Mater. Chem. B, 2015, 3, 286-295.

22 X. Liu, X. Lin, M. Wu, R. Lin, B. Li and J. Liu, J. Mater. Chem. $B, 2016,4,4119-4129$.

23 R. A. Smith, C. M. Porteous, A. M. Gane and M. P. Murphy, Proc. Natl. Acad. Sci. U. S. A., 2003, 100, 5407-5412.

24 Y. L. Chang, X. D. Li, X. G. Kong, Y. Li, X. M. Liu, Y. L. Zhang, L. P. Tu, B. Xue, F. Wu, D. B. Cao, H. Y. Zhao and H. Zhang, J. Mater. Chem. B, 2015, 3, 8321-8327. 
25 B. F. Zhang, L. Xing, P. F. Cui, F. Z. Wang, R. L. Xie, J. L. Zhang, M. Zhang, Y. J. He, J. Y. Lyu, J. B. Qiao, B. A. Chen and H. L. Jiang, Biomaterials, 2015, 61, 178-189. 26 D. R. Green and J. C. Reed, Science, 1998, 281, 1309-1312.

27 R. M. Kluck, E. BossyWetzel, D. R. Green and D. D. Newmeyer, Science, 1997, 275, 1132-1136.

28 K. M. Tainton, M. J. Smyth, J. T. Jackson, J. E. Tanner, L. Cerruti, S. M. Jane, P. K. Darcy and R. W. Johnstone, Cell Death Differ., 2004, 11, 1028-1037.
29 J. A. MacKay, M. Chen, J. R. McDaniel, W. Liu, A. J. Simnick and A. Chilkoti, Nat. Mater., 2009, 8, 993-999.

30 K. H. Min, K. Park, Y. S. Kim, S. M. Bae, S. Lee, H. G. Jo, R. W. Park, I. S. Kim, S. Y. Jeong, K. Kim and I. C. Kwon, J. Controlled Release, 2008, 127, 208-218.

31 Y. Ding, J. Han, B. Tian, J. Han, J. Zhang, H. Zheng, Y. Han and M. Pei, Int. J. Pharm., 2014, 477, 187-196. 